\title{
@creative
}

ISSN 1855-3966 (printed edn.), ISSN 1855-3974 (electronic edn.)

\author{
ARS MATHEMATICA CONTEMPORANEA 17 (2019) 255-270 \\ https://doi.org/10.26493/1855-3974.1560.a43 \\ (Also available at http://amc-journal.eu)
}

\section{Tetrahedral and pentahedral cages for discs*}

\author{
Liping Yuan \\ College of Mathematics and Information Science, Hebei Normal University, \\ 050024 Shijiazhuang, P.R. China \\ Hebei Key Laboratory of Computational Mathematics and Applications, \\ 050024 Shijiazhuang, P.R. China
}

\section{Tudor Zamfirescu ${ }^{\dagger}$}

Fachbereich Mathematik, Technische Universität Dortmund, 44221 Dortmund, Germany Institute of Mathematics "Simion Stoilow", Roumanian Academy, Bucharest, Roumania College of Mathematics and Information Science, Hebei Normal University, 050024 Shijiazhuang, P.R. China

Received 21 December 2017, accepted 21 April 2019, published online 14 October 2019

\begin{abstract}
This paper is about cages for compact convex sets. A cage is the 1-skeleton of a convex polytope in $\mathbb{R}^{3}$. A cage is said to hold a set if the set cannot be continuously moved to a distant location, remaining congruent to itself and disjoint from the cage.

In how many "truly different" positions can (compact 2-dimensional) discs be held by a cage? We completely answer this question for all tetrahedra. Moreover, we present pentahedral cages holding discs in a large number (57) of positions.
\end{abstract}

Keywords: Tetrahedral cages, pentahedral cages, discs.

Math. Subj. Class.: $52 B 10$

\section{Introduction}

A cage is the 1 -skeleton of a (convex) polytope in $\mathbb{R}^{3}$. If $P$ is the polytope, the cage is denoted by cage $(P)$. A cage $G$ is said to hold a compact set $K$ with $G \cap \operatorname{int} K=\emptyset$, if no rigid continuous motion can bring $K$ in a position far away without int $K$ meeting $G$ on its way. (Here, int $K$ means the interior of $K$ in its affine hull.) A compact 2-dimensional ball in $\mathbb{R}^{3}$ will be called a disc.

\footnotetext{
*This work is supported by NSF of China (No. 11871192, No. 11471095).

${ }^{\dagger}$ The author was partly supported by the GDRI ECO-Math.

E-mail addresses: lpyuan@ hebtu.edu.cn (Liping Yuan), tuzamfirescu@gmail.com (Tudor Zamfirescu)
} 
Not that long ago, the subject of holding (3-dimensional) balls in cages has been treated by Coxeter [6], Besicovitch [4], Aberth [1] and Valette [12].

In this paper we hold discs instead of balls. The question we ask is about the number of positions of the discs held.

We investigate the capability of the 1-skeleton of the regular tetrahedron as a cage to hold discs. Then, we consider the capability of the 1-skeleton of an arbitrary tetrahedron to hold discs, and discuss in detail the dependence on the shape of the tetrahedron. Finally, we also consider the two combinatorial types of pentahedral cages.

The related phenomenon of holding a convex body using a circle was investigated in [2, 3, 13]. For other related results, see [9, 10, 14, 15].

For distinct $x, y \in \mathbb{R}^{3}$, let $\overline{x y}$ be the line through $x, y$ and $x y$ the line-segment from $x$ to $y$. We denote by $\Pi_{x y}$ the plane through $x$ orthogonal to $\overline{x y}$, and by $\Pi_{x y}^{+}$the closed half-space not containing $y$, determined by $\Pi_{x y}$.

For $M \subset \mathbb{R}^{3}, \bar{M}$ denotes its affine hull, int $M$ and bd $M$ denote its interior and boundary in the topology of $\bar{M}$, and $\operatorname{diam} M=\sup _{x, y \in M}\|x-y\|$. A line-segment $x y$ with $\{x, y\} \subset M$ and $\|x-y\|=\operatorname{diam} M$ is called a diameter of $M$. Also, conv $M$ denotes the intersection of all convex sets including $M$.

For $x_{1}, x_{2}, \ldots, x_{k} \in \mathbb{R}^{3}, x_{1} x_{2} \cdots x_{k}$ means $\operatorname{conv}\left\{x_{1}, x_{2}, \ldots, x_{k}\right\}$. For non-collinear elements $x, y, z \in \mathbb{R}^{3}$, let $C(x y z) \subset \overline{x y z}$ be the circle passing through $x, y, z$, and let $o_{x y z}$ be its centre. Put $D(x y z)=\operatorname{conv} C(x y z)$. We denote by $\widehat{x y z}$ the angle of $x y z$ at $y$, and by $\angle x y z$ its measure.

A face of a cage $G$ is a 2-dimensional face of the polytope conv $G$.

The $d$-dimensional compact unit ball (centred at $\mathbf{0}$ ) is $B_{d}$, and bd $B_{d}=S_{d-1}(d \geq 2$ ).

Also, we denote by $\lambda$ the 1-dimensional Hausdorff measure (length).

Problem 1.1. Let $\mathcal{G}(K)$ be the space of all cages in $\mathbb{R}^{3}$ holding the compact set $K$. Determine

$$
L(K)=\inf _{G \in \mathcal{G}(K)} \lambda G,
$$

for various sets $K$.

This problem, in line with the work of Coxeter, Besicovitch, Aberth and Valette, will not be addressed in this paper, but in [8].

For any cage $G$, let $\mathcal{D}(G)$ be the space of all discs held by $G$, endowed with the Pompeiu-Hausdorff metric.

Let $\mathcal{D}_{r}(G)$ be the set of all discs in $\mathcal{D}(G)$ of radius at least $r$. (Notice that the term "radius" is used for both the distance and the line-segment from the centre to a point of the relative boundary.) Assume that, for some component $\mathcal{E}$ of $\mathcal{D}_{r}(G)$ and any number $s>r$, $\mathcal{D}_{s}(G) \cap \mathcal{E}$ is connected or empty. We call such a component $\mathcal{E}$ an end-component of $\mathcal{D}(G)$. If $n$ is the maximal number of pairwise disjoint end-components of $\mathcal{D}(G)$, we say that $G$ holds $n$ discs.

In fact, intuitively, $G$ does not hold $n$ pairwise disjoint discs simultaneously; merely there are $n$ different positions at which, separately, a disc can be held.

Let the component $\mathcal{E}$ of $\mathcal{D}_{r}(G)$ be an end-component of $\mathcal{D}(G)$. Put $\sigma(\mathcal{E})=\sup \{s$ : $\left.\mathcal{D}_{s}(G) \cap \mathcal{E} \neq \emptyset\right\}$. Choose an increasing sequence $\left\{s_{n}\right\}_{n=1}^{\infty}$ of real numbers satisfying $s_{n}>r$ and $\lim _{n \rightarrow \infty} s_{n}=\sigma(\mathcal{E})$. Consider a disc $D_{n} \in \mathcal{D}_{s_{n}}(G)$ for each $n$.

If $\left\{D_{n}\right\}_{n=1}^{\infty}$ converges to some disc $D(\mathcal{E})$ independent of the choice of the numbers $s_{n}$ and discs $D_{n}$, we call $D(\mathcal{E})$ the limit disc of $\mathcal{E}$. Several end-components may have the same limit disc. 
If the limit disc of an end-component $\mathcal{E}$ lies in the plane of a face $F$ of conv $G$, we say that $G$ holds a disc at the face $F$. For each end-component, we have a disc held, even if the limit discs coincide. So, a cage may hold several discs at the same face. Also, if a face $F$ is not triangular, several distinct limit discs can be coplanar with $F$.

Inspired by an earlier version of the present paper, Montejano and Zamfirescu [11] raised the following questions.

Problem 1.2. Does a cage holding 7 discs exist?

Problem 1.3. How many discs can be held by a pentahedral cage?

We give here an affirmative answer to Problem 1.2, establish the precise minimum and find a lower bound for the maximum number of discs that a pentahedral cage can hold.

For a cage which is not tetrahedral it is possible that a disc is held, but not at a face. Such a case we shall meet for a pentahedral cage admitting a limit disc (of some end-component) circumscribed to a triangle which is not a face of the pentahedron, but has vertices among those of the cage. For arbitrary polyhedral cages even the following is possible.

Proposition 1.4. There exist cages $G$ admitting a limit disc not coplanar with any vertex of conv $G$.

Proof. Consider a regular icosagon $\Delta=a_{1} a_{2} \cdots a_{20} \subset H$ inscribed in $S_{1}$, where $H=$ $\{(x, y, z): z=0\}$ and $S_{1}$ is the unit circle in $H$. Let $\varepsilon>0$ and $\tau=(0,0, \varepsilon)$. Let $\nu>0$.

Put

$$
b_{i}=\left\{\begin{array}{lll}
(1+\nu) a_{i}+\tau & \text { for } i \not \equiv 3 & (\bmod 4) \\
(1-\nu) a_{i}+\tau & \text { for } i \equiv 3 & (\bmod 4)
\end{array}\right.
$$

and

$$
c_{i}=\left\{\begin{array}{lll}
(1+\nu) a_{i}-\tau & \text { for } i \neq \equiv 1 & (\bmod 4) \\
(1-\nu) a_{i}-\tau & \text { for } i \equiv 1 & (\bmod 4)
\end{array}\right.
$$

For $\nu$ small enough, $\Delta_{b}=b_{1} b_{2} \cdots b_{20}$ and $\Delta_{c}=c_{1} c_{2} \cdots c_{20}$ are convex icosagons. The polytope $P=\operatorname{conv}\left(\Delta_{b} \cup \Delta_{c}\right)$ has 42 faces including $\Delta_{b}$ and $\Delta_{c}$. We claim that $B_{2}$ is a limit disc of cage $(P)$.

Indeed, note that the circle $S_{1}$ meets cage $(P)$ at the vertices $a_{1}, a_{3}, \ldots, a_{19}$ of $\Delta$ only. Assume that some unit disc $D$ distinct from $B_{2}$ but close to it satisfies cage $(P) \cap \operatorname{int} D=$ $\emptyset$. Let the ellipse $E$ be the orthogonal projection of $D$ onto $H$ and let $x y$ be the long axis of $E$ (or any diameter if $E$ is a circle). Since $\|x-y\|=2$, one of these end-points, say $x$, is on $S_{1}$ or outside $B_{2}$. Let $x^{\prime}, y^{\prime}$ be the points of $D$ with projections $x, y$, respectively. Since $\overline{x^{\prime} y^{\prime}}$ is parallel to $H$, it is included in (at least) one of the half-spaces

$$
H^{+}=\{(x, y, z): z \geq 0\}, \quad H^{-}=\{(x, y, z): z \leq 0\} .
$$

Suppose without loss of generality that $\overline{x^{\prime} y^{\prime}} \subset H^{-}$. Then, at least one of the half-discs of $D$ determined by $x^{\prime} y^{\prime}$, say $D^{\prime}$, entirely lies in $H^{-}$.

The intersection $\left\{x^{*}\right\}=\mathbf{0} x \cap S_{1}$ lies on $S_{1}$ between two consecutive vertices of the regular pentagon $a_{1} a_{5} a_{9} a_{13} a_{17}$, or coincides with one of them, say $x^{*} \in \widehat{a_{1} a_{5}}$. Therefore, since $D \neq B_{2}, D^{\prime}$ cuts either $a_{1} c_{1}$ or $a_{5} c_{5}$, which yields int $D \cap \operatorname{cage}(P) \neq \emptyset$, and this contradicts our assumption. 


\section{Tetrahedral cages}

Consider a regular tetrahedron. If its edge-length is 1 , then the circle circumscribed to a face has radius $1 / \sqrt{3}$. So, a slightly enlarged tetrahedral cage $T$ will hold the disc $(1 / \sqrt{3}) B_{2}$. Clearly, at each face there is such a disc.

In fact there are many discs close to $(1 / \sqrt{3}) B_{2}$, held by $T$, lying in the same component of $\mathcal{D}_{(1 / \sqrt{3})}(T)$. The space $\mathcal{D}_{(1 / \sqrt{3})}(T)$ has 4 components analogous to the component of $(1 / \sqrt{3}) B_{2}$, one corresponding to each face of $T$. The limit disc of each component is the disc circumscribed to the respective face.

The following lemma is easily verified by the reader.

Lemma 2.1. If a polytopal cage holds a disc at some triangular face, then that triangle is acute.

A face being an acute triangle is, however, no guarantee that the disc described above (lying over the face) is held there. Whether it can move away from that face or not, obviously depends on the angle between the edges of the polytope adjacent but not belonging to that face and the corresponding radii of the circumscribed circle of the face.

Lemma 2.2. If a face of a tetrahedral cage is an acute triangle, then at least one disc is held at that face.

Proof. Let $a b c$ be the given acute face, and $o$ the centre of $C(a b c)$. Consider the half-spaces $\Pi_{a o}^{+}, \Pi_{b o}^{+}, \Pi_{c o}^{+}$. As the intersection of these half-spaces is void, there is no point $x \in \mathbb{R}^{3}$ for which all angles $\widehat{x a o}, \widehat{x b o}, \widehat{x c o}$ are non-acute. Assume $\angle d a o<\pi / 2$. Now take a disc (slightly smaller than $D(a b c)$ ) over $a b$ and $a c$, but below $b c$ (see Figure 1). This disc is held by the cage.

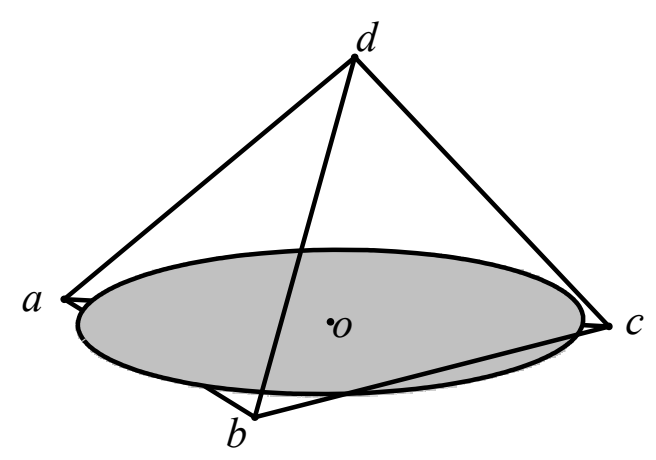

Figure 1: Cage holding a disc.

Lemma 2.3. If a tetrahedral cage has an acute face, then it has one, two, or four discs held at that face.

Proof. Keep the notation of the preceding proof. The kind of disc held by the cage in the previous proof requires an angle like $\widehat{d a o}$ to be acute. The existence of a second such angle, say $\widehat{d b o}$, provides a second such disc. If at least one such angle, say $\widehat{d c o}$, is not acute, then 
any disc lying over the face $a b c$ can move away from the face. If all three angles $\widehat{d a o}$, $\widehat{d b o}, \widehat{d c o}$ are acute, then not only the three discs partly lying below some edge of $a b c$ are held, but also the disc lying completely over the face $a b c$, whence the conclusion of the lemma.

Theorem 2.4. The regular tetrahedral cage holds 16 discs.

Proof. The last case of the proof of Lemma 2.3 applies at all faces. By Proposition 2.5 below, there is no other disc held by the cage.

Tetrahedral cages cannot display the situation in Proposition 1.4.

Proposition 2.5 (Fruchard [7]). In any tetrahedral cage, each limit disc is at some face.

With the author's permission, we reproduce here his proof, for the reader's convenience.

Proof. Let $a b c d$ be a non-degenerate tetrahedron, $G=\operatorname{cage}(a b c d)$, and assume $D$ is a limit disc which is not at a face. To fix ideas, we assume that $D$ is the unit disc $B_{2}$ in the horizontal plane $H=\{(x, y, z): z=0\}$ of $\mathbb{R}^{3}$.

It is an easy task to exclude that some vertex of $G$ lies in the plane of $D$. Furthermore, it is easily seen that $D$ meets four edges of $G$, say $a b, b c, c d$, and $d a$, with $a$ and $c$ above $D$, and $b$ and $d$ below $D$. Two of these edges have to pass above $D$ and two below, and they must alternate, say $a b$ and $c d$ above, $b c$ and $d a$ below. Let $e \in a b \cap D, f \in b c \cap D$, $g \in c d \cap D$, and $h \in d a \cap D$, see Figure 2 .

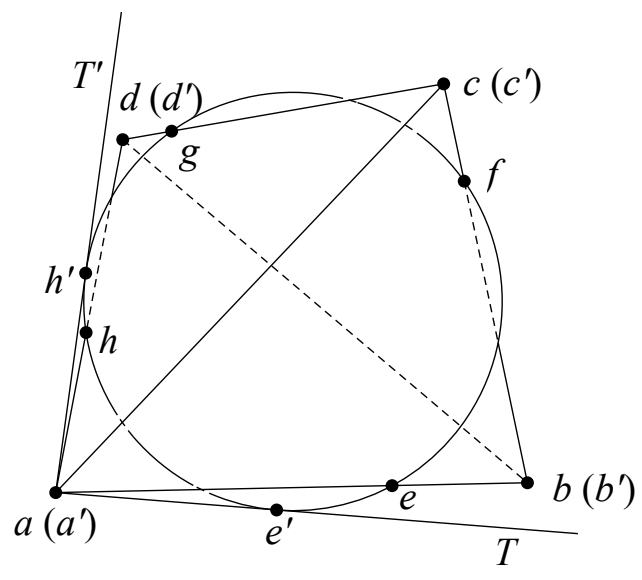

Figure 2: Proof of Proposition 2.5.

Let $a^{\prime}, b^{\prime}, c^{\prime}$ and $d^{\prime}$ be the orthogonal projections of $a, b, c, d$ on $H$. Then, we have

$$
\frac{\left\|a^{\prime}-e\right\|}{\left\|b^{\prime}-e\right\|}=\frac{\|a-e\|}{\|b-e\|}=\frac{z_{a}}{\left|z_{b}\right|},
$$

where $z_{a}$ is the third coordinate of $a$. Using the analogous formulae for the other three sides of the quadrilateral $a^{\prime} b^{\prime} c^{\prime} d^{\prime}$, we obtain

$$
\frac{\left\|a^{\prime}-e\right\|}{\left\|b^{\prime}-e\right\|} \frac{\left\|b^{\prime}-f\right\|}{\left\|c^{\prime}-f\right\|} \frac{\left\|c^{\prime}-g\right\|}{\left\|d^{\prime}-g\right\|} \frac{\left\|d^{\prime}-h\right\|}{\left\|a^{\prime}-h\right\|}=\frac{z_{a}}{\left|z_{b}\right|} \frac{\left|z_{b}\right|}{z_{c}} \frac{z_{c}}{\left|z_{d}\right|} \frac{\left|z_{d}\right|}{z_{a}}=1 .
$$


As we show below, this is impossible. From $a^{\prime}$, draw the two tangent lines to $D, T$ toward $b$ and $T^{\prime}$ toward $d$. Let $e^{\prime} \in T \cap D$ (hence on the same side as $e$ ) and $h^{\prime} \in T^{\prime} \cap D$. Because $a b$ is above $D$, we have $\|a-e\|>\left\|a^{\prime}-e^{\prime}\right\|$; in the same manner, $d a$ is below $D$, hence $\left\|a^{\prime}-h\right\|<\left\|a^{\prime}-h^{\prime}\right\|$. Then, $\left\|a^{\prime}-e^{\prime}\right\|=\left\|a^{\prime}-h^{\prime}\right\|$ implies $\frac{\left\|a^{\prime}-e\right\|}{\left\|a^{\prime}-h\right\|}>1$. Similarly, one has $\frac{\left\|b^{\prime}-f\right\|}{\left\|b^{\prime}-e\right\|}, \frac{\left\|c^{\prime}-g\right\|}{\left\|c^{\prime}-f\right\|}$ and $\frac{\left\|d^{\prime}-h\right\|}{\left\|d^{\prime}-g\right\|}$ all larger than 1 , contradicting equation (2.1).

Lemma 2.6. If, for $a, b, c, x, o \in \mathbb{R}^{3}, \angle a x b \leq \pi / 2, \angle c x a<\pi / 2$ and o lies in the relative interior of bxc, then $\angle a x o<\pi / 2$.

The proof (using for example the basic properties of the scalar product) is left to the reader.

Theorem 2.7. There are tetrahedral cages holding exactly $n$ discs, for every $n \leq 16$ except for $n \in\{7,9,11,13,14,15\}$, and there is no such cage for any other $n$.

Proof. Separately, every number $n$ of held discs can be realized at a face, if $n \in\{0,1,2,4\}$, by Lemma 2.3. We have to show that a global realization is possible, for each of the $n$ 's from the statement. Moreover, we must show the impossibility of a realization in all other cases.

We keep in mind that limit discs can only be at faces, by Proposition 2.5.

Throughout this proof, $o$ will denote the centre of $C(a b c)$.

Case $\boldsymbol{n}=0$ : Take the face $a b c$ to have an obtuse angle at $a$, take a point $d^{\prime}$ in the relative interior of its height at $a$, and consider a point $d$ close to $d^{\prime}$ and having $d^{\prime}$ as orthogonal projection on $\overline{a b c}$. Then the tetrahedral cage cage $(a b c d)$ has all faces obtuse. Now use Lemma 2.1.

Case $\boldsymbol{n}=1$ : Take now the face $a b c$ to be an acute triangle and consider $o$. For any point

$$
d \in \Pi_{a o}^{+} \cap \Pi_{b a}^{+} \backslash \overline{a b c}
$$

the triangles $a b d, b c d, c a d$ are obtuse or right. See Figure 3.

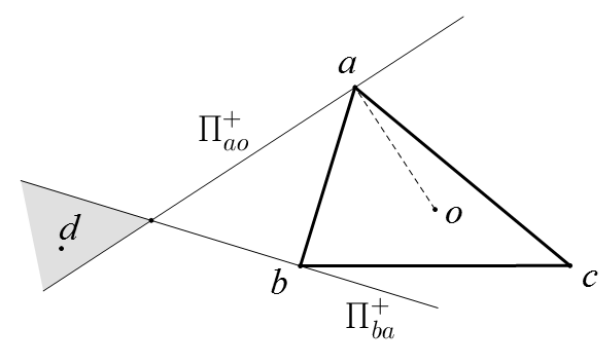

Figure 3: Case $n=1$.

Moreover, only one of the angles $\widehat{o a d}, \widehat{o b d}, \widehat{o c d}$ is acute, namely the latter. Thus, cage $(a b c d)$ holds exactly one disc (at the face $a b c$ ), as described in the proof of Lemma 2.2.

Case $\boldsymbol{n}=2$ : Let again $a b c$ be acute, and choose

$$
d \in \Pi_{a c}^{+} \cap \Pi_{b a}^{+} \backslash\left(\Pi_{a o}^{+} \cup \overline{a b c}\right) .
$$


In this way $a b d, b c d, c a d$ are still non-acute, but now precisely two of the angles $\widehat{o a d}, \widehat{o b d}$, $\widehat{o c d}$ are acute, namely the first and the last (see Figure 4). Thus, two discs are held, both at the face $a b c$.

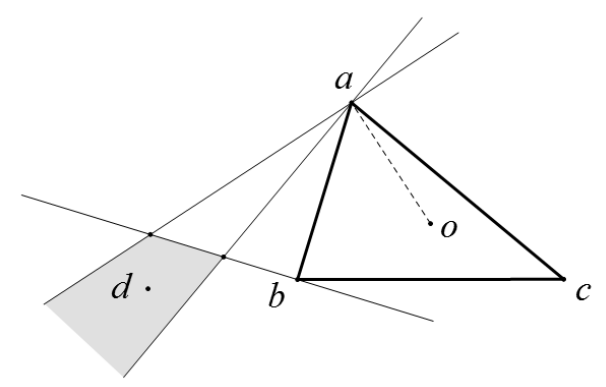

Figure 4: Case $n=2$.

Case $\boldsymbol{n}=3$ : Take $a b c$ acute, as before. Choose

$$
d \in \Pi_{a c}^{+} \cap \Pi_{b o}^{+} \backslash\left(\Pi_{b a}^{+} \cup \Pi_{a o}^{+} \cup \overline{a b c}\right) .
$$

Now, the triangles $b c d$ and $c a d$ are non-acute, while the triangles $a b c$ and $a b d$ are acute. See Figure 5.

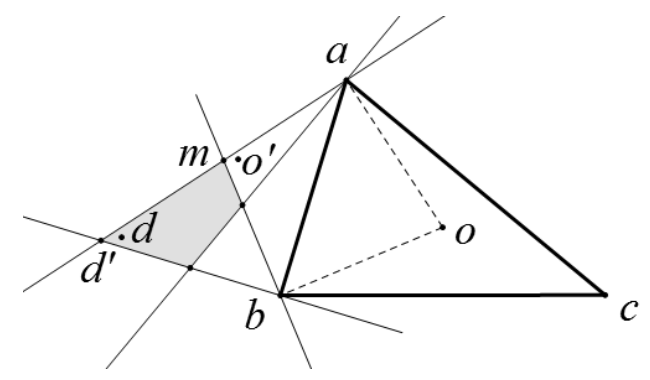

Figure 5: Case $n=3$. at $a b c$.

Regarding $a b c, \angle o a d<\pi / 2, \angle o b d \geq \pi / 2, \angle o c d<\pi / 2$, whence two discs are held

Regarding $a b d$, let $\left\{d^{\prime}\right\}=\Pi_{a o} \cap \Pi_{b a} \cap \overline{a b c}$, and denote by $m$ the midpoint of $a d^{\prime}$. Then $\angle o a m=\angle o b m=\pi / 2$. Hence, $\angle c a m>\pi / 2$ and $\angle c b m>\pi / 2$. If $d$ is chosen close to $d^{\prime}$ (and in the already assigned region), then the centre $o^{\prime}$ of $C(a b d)$ is close to $m$, and we also have $\angle c a o^{\prime}>\pi / 2$ and $\angle c b o^{\prime}>\pi / 2$. Doubtlessly $\angle c d o^{\prime}<\pi / 2$, whence there is precisely one disc held by cage $(a b c d)$ at $a b d$.

Case $\boldsymbol{n}=4$ : Let the face $a b c$ be an equilateral triangle of centre $o$. Choose $d \notin \overline{a b c}$ close to $o$. Thus, the triangles $d a b, d b c$ and $d c a$ are obtuse. See Figure 6. By Lemma 2.1, no disc is held at any of the faces $d a b, d b c, d c a$.

Since $\angle o a d, \angle o b d$ and $\angle o c d$ are close to 0 , cage $(a b c d$ ) holds exactly 4 discs at $a b c$ (see the proof of Lemma 2.3). 


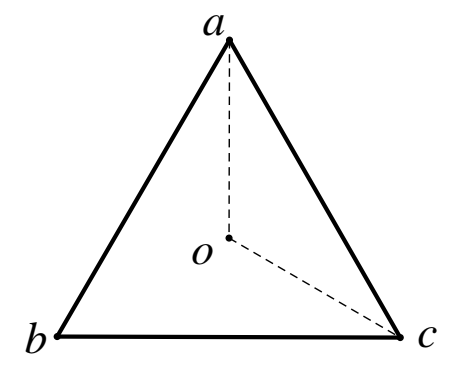

Figure 6: Case $n=4$.

Case $n=5$ : Let $a^{\prime} b e c$ be a square. Choose $a, d^{\prime} \in \overline{a^{\prime} e}$ such that $a, a^{\prime}, e, d^{\prime}$ lie in this order on their line, with $\left\|a-a^{\prime}\right\|$ small and $\left\|e-d^{\prime}\right\|=\|b-e\|$. See Figure 7 . Then

$$
\angle a b e=\angle a c e>\pi / 2 \text { and } \angle o b d^{\prime}=\angle o c d^{\prime}<\pi / 2 .
$$

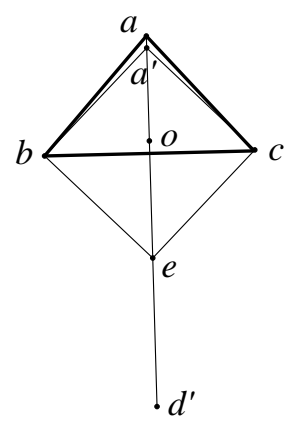

Figure 7: Case $n=5$.

Rotate slightly $d^{\prime}$ about $\overline{b c}$ up to a new position $d$. Then still

$$
\angle a b o^{\prime}=\angle a c o^{\prime}>\pi / 2 \quad \text { and } \quad \angle o b d=\angle o c d<\pi / 2,
$$

where $o^{\prime}$ is the centre of $C(b c d)$.

Also, notice that $\angle a d o^{\prime}$ and $\angle o a d$ are small.

The triangles $a b c$ and $b c d$ are acute, $a b d$ and $a c d$ obtuse. The inequalities above imply that one disc is held by cage $(a b c d)$ at $b c d$, and four discs at $a b c$.

Case $\boldsymbol{n}=6$ : Take an equilateral triangle $a b c$, and choose $a^{\prime} \in a o$ such that $\angle b a^{\prime} c<\pi / 2$. Let $d \in \mathbb{R}^{3} \backslash \overline{a b c}$ be close to $a^{\prime}$, such that $a^{\prime}$ is its orthogonal projection on $\overline{a b c}$. Then 4 discs are held at $a b c$ and 2 discs at $b c d$ (see the proof of Lemma 2.3).

Case $n \in\{\mathbf{7}, \mathbf{9}, \mathbf{1 1}, \mathbf{1 3}\}$ : By Lemma 2.3, in order to obtain exactly 7 discs held by cage $(a b c d)$, there are 3 possibilities for the number of discs held at each face: $2,2,2,1$, or $4,1,1,1$, or $4,2,1,0$. 
To obtain exactly 9 discs held by cage $(a b c d)$, there are 2 possibilities for the number of discs held at each face: $4,2,2,1$, or $4,4,1,0$.

To obtain exactly 11 discs held, there is just one possibility for the number of discs held at each face: $4,4,2,1$.

Similarly for 13 discs held: $4,4,4,1$.

In each of these 7 scenarios, there exists a face at which exactly one disc is held and at most one face at which no disc is held. We prove this to be impossible to realize.

Suppose it is realized. Then at most one of the 12 angles (of the 4 triangles), say $\widehat{a c d}$, is non-acute. Consequently, the triangles $a b c, b c d$ and $a b d$ are acute, and all angles at $a, b, d$ are acute, too.

By Lemma 2.6, $\angle$ oad $<\pi / 2$ and $\angle o b d<\pi / 2$; thus, at least two discs are held at $a b c$. Similarly, at least two discs are held at $b c d$. At $a b d$ exactly four discs are held, as all cage angles at $a, b, d$ are acute.

Now, if acd is not acute, no disc is held there. If acd is acute, then each face behaves like $a b d$, i.e. 4 discs are held at each face. Hence, at no face exactly one disc is held.

Case $\boldsymbol{n}=8$ : Take two coplanar equilateral triangles $a b c$ and $b c d^{\prime}$, and then slightly rotate the latter about $\overline{b c}$ to reach a new position $b c d$. Then the angles oad, obd and ocd are acute, whence cage $(a b c d)$ holds 4 discs at $a b c$. By symmetry, it also holds 4 discs at $b c d$. As $a b d$ and $a c d$ are obtuse triangles, there are no further discs held by cage $(a b c d)$.

Case $\boldsymbol{n}=10$ : Let the triangle $a b c$ be equilateral, and $d^{\prime}$ be close to $a$, such that $\|a-c\|=$ $\left\|c-d^{\prime}\right\|$ and $a c \cap o d^{\prime} \neq \emptyset$. Let $o^{\prime}$ be the centre of $C\left(b c d^{\prime}\right)$, and $o^{\prime \prime}$ the centre of $C\left(a c d^{\prime}\right)$. (See Figure 8.)

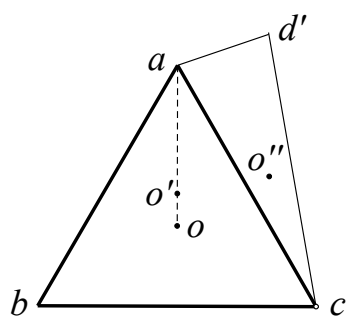

Figure 8: Case $n=10$.

Clearly,

$$
\angle d^{\prime} a o>\pi / 2, \quad \angle d^{\prime} b o<\pi / 2, \quad \angle d^{\prime} c o<\pi / 2 .
$$

Also,

$$
\angle a d^{\prime} o^{\prime}<\pi / 2, \quad \angle a b o^{\prime}<\pi / 2, \quad \angle a c o^{\prime}<\pi / 2
$$

and

$$
\angle b a o^{\prime \prime}<\pi / 2, \quad \angle b c o^{\prime \prime}<\pi / 2, \quad \quad \angle b d^{\prime} o^{\prime \prime}<\pi / 2 .
$$

By rotating a little $d^{\prime}$ about $\overline{a c}$, the above angles don't change much, and the inequalities remain valid. Let $d$ be the new position of $d^{\prime}$. So, there are 4 discs held at $b c d, 4$ at $a c d$, just 2 at $a b c$, and none at $a b d$, as $\angle b a d>\pi / 2$. 
Case $\boldsymbol{n}=12$ : Let cage $(a b c d)$ have three acute triangular faces and a right triangle $a b c$ as fourth face, with $\angle b a c=\pi / 2$. By Lemma 2.6,

$$
\angle b a o<\pi / 2, \quad \angle b c o<\pi / 2, \quad \angle b d o<\pi / 2,
$$

whence 4 discs are held at $c d a$. Analogously, $a b c d$ holds 4 discs at each of the faces $d a b$, $b c d$. Of course, no disc is held at $a b c$.

Case $\boldsymbol{n}=14$ : Suppose cage $(a b c d)$ holds 4, 4, 4, 2 discs at the four faces, which is the only possibility of reaching the total number of 14 . Then all triangles are acute. By Lemma 2.6, $\angle$ oad $<\pi / 2, \angle o b d<\pi / 2, \angle o c d<\pi / 2$, whence there are 4 discs held at $a b c$. This applies to every face. Hence, at no face the number of discs held is 2 .

Case $\boldsymbol{n}=15$ : Impossible as sum of four integers from $\{0,1,2,4\}$.

Case $\boldsymbol{n}=16$ : The regular tetrahedron realizes this, see Theorem 2.4.

If we briefly say that the cage $G$ holds $n$ unit discs, this means that $G$ holds $n$ discs, i.e. the maximal number of pairwise disjoint end-components is $n$, and $\sigma(\mathcal{E})$ does not depend on the chosen end-component $\mathcal{E}$.

One may ask the question: how many unit discs can a tetrahedral cage hold? We shall not deepen this question here, only make some remarks.

Trivially, by Theorem 2.7, there is a cage holding 1 unit disc.

In the proof for $n=2$, both discs held by the cage were at the same face, so they had the same size. Similarly, Theorem 2.4 shows that the regular tetrahedral cage holds 16 unit discs.

The proof for $n=3$ provides two discs of same size, and a third disc of a possibly different size. A more concrete construction is needed. We do this here, using the notation from the proof of Theorem 2.7, case $n=3$.

The acute triangle $a b c$ will be taken such that $\angle a c b=\frac{\pi}{4}$, which implies $\angle o a b=$ $\angle o b a=\pi / 4$. Now, the two circles $C(a b c)$ and $C\left(a b d^{\prime}\right)$ are congruent.

Let $\Theta$ be the torus obtained by rotating $C\left(a b d^{\prime}\right)$ about $\overline{a b}$. By choosing $d \in \Theta \backslash\left(\Pi_{b a}^{+} \cup\right.$ $\left.\Pi_{a o}^{+} \cup \overline{a b c}\right)$, still close to $d^{\prime}$, we get $C(a b d)$ and $C(a b c)$ congruent.

For the regular tetrahedral cage $T$ of unit side-length, any disc held has radius at least $1 / 2$.

Altogether $T$ holds 16 discs, by Theorem 2.7. In fact, for any $r \in[3 \sqrt{2} / 8, \sqrt{3} / 3]$, $\mathcal{D}_{r}(T)$ has 16 components. What happens for smaller $r$ ?

Theorem 2.8. Let $T$ be the regular tetrahedral cage of unit side-length. For any $r \in$ $[1 / 2,3 \sqrt{2} / 8], \mathcal{D}_{r}(T)$ has 4 components.

Proof. A disc $D$ in $\mathcal{D}_{r}(T)$ above $a b c$ can be rotated about an axis parallel and close to $a b$ without meeting $c d$ until it reaches a position close to $a b d$, above $a d$ and $b d$, but below $a b$ (seeing now $a b d$ as horizontal, with $T$ above it).

The rotation of the disc $D$ can also be performed about an axis close to $b c$, or $b d$, and so we obtain a third and a fourth disc in the same component as $D$. This means that a group of 4 discs held by $T$ among the 16 analogous to those mentioned in Theorem 2.4 belong to the same component of $\mathcal{D}_{r}(T)$. As we have 4 such groups, the conclusion of the theorem follows.

Theorem 2.8 provides illuminating examples of components which are not end-components of $\mathcal{D}(T)$. 


\section{Pentahedral cages}

The convex pentahedra are of two combinatorial types: the pyramid over a quadrilateral and the triangular prism. We do not aim at finding all possible numbers of discs which can be held by pentahedral cages, as we did for tetrahedra. We restrict the otherwise lengthy analysis to the most interesting problem about the maximal number of discs which can be held.

We start with the question: How many discs can a pentahedral cage hold at a face? We know the answer if the face is triangular by adapting the analysis from the tetrahedral case to this new situation: $0,1,2$, or 4 . This is seen like in Lemma 2.3, with the difference that the case of 0 discs may now occur, even if the triangle is acute. For our pentahedra we need the answer for quadrilateral faces, too.

Let $Q=a b c d$ be a quadrilateral (bottom) face of a polytope $P$, and assume that each vertex of $Q$ has degree 3 in $P$. (This is so in pentahedra.) Each diagonal of $Q$ divides it into two triangles. These four triangles cannot all be acute, at least one must be non-acute. Let $a^{\prime}$ be the vertex of $P$, neighbour of $a$, different from $b, d$. Also, consider the analogous vertices $b^{\prime}, c^{\prime}, d^{\prime}$. (Some of these vertices may coincide.)

An exhaustive investigation would have to consider several cases. But this is not our intention. As an example, we treat the case when $a, b, c, d$ are cocyclic. Assume $a b c$ and $a b d$ are acute. Obviously, both $d \in D(a b c), c \in D(a b d)$. Moreover, the inequalities $\angle d a o_{a b c}<\pi / 2, \angle d b o_{a b c}<\pi / 2$ and $\angle d c o_{a b c}<\pi / 2$ are satisfied. Thus, if all inequalities $\angle a^{\prime} a o_{a b c}<\pi / 2, \angle b^{\prime} b o_{a b c}<\pi / 2, \angle c^{\prime} c o_{a b c}<\pi / 2$ are valid, then a disc can be held over $a b, b c, c d$, and $d a$, or over 3 of them and under the fourth, or over $a b, b c$ and under $c d, d a$, or over $d a, a b$ and under $b c, c d$, which gives 7 possibilities in total.

In case $a, b, c, d$ are not cocyclic, more discs can be held at $Q$.

Lemma 3.1. If the triangles $a b c, a b d, b c d$ and the angles

$$
\begin{array}{lllll}
\widehat{a d o_{b c d}}, & \widehat{c d o_{a b d}}, & \widehat{a^{\prime} a o_{a b d}}, & \widehat{a^{\prime} a o_{a b c}}, & \widehat{b^{\prime} b o_{a b d}}, \\
\widehat{b^{\prime} b o_{b c d}}, & \widehat{c^{\prime} c O_{a b c}}, & \widehat{c^{\prime} c O_{b c d}}, & \widehat{d^{\prime} d o_{a b d}}, & \widehat{d^{\prime} d o_{b c d}},
\end{array}
$$

are all acute, then 13 discs are held at $Q=$ abcd.

Proof. First of all, by Lemma 2.6, $\angle b^{\prime} b o_{a b d}<\pi / 2$ and $\angle b^{\prime} b o_{b c d}<\pi / 2$ imply $\angle b^{\prime} b o_{a b c}<\pi / 2$.

Now, considering $a b d$, a disc is held above all four edges, another one is held under $a b$ and above the other three, yet another disc under $a d$ and above all others, a fourth disc under $b c$ and $c d$ and above $a b$ and $d a$, a fifth under $b c$ and above all others, and a sixth under $c d$ and above the remaining edges.

Analogously, considering $b c d$, we find other six discs held.

Moreover, considering $a b c$, one more disc is held, namely under $c d$ and $d a$ and above $a b$ and $b c$.

Lemma 3.2. There are maximally 13 discs held at abcd.

Proof. It is quickly seen that, in all other cases concerning the angles mentioned at Lemma 3.1, the number of discs held is smaller than 13 .

In conclusion, at any quadrilateral face of a polytopal cage, at most 13 discs can be held, and this only if several angle inequalities are satisfied. If the polytope is a prism, the following holds. 
Lemma 3.3. Let $a b c a^{*} b^{*} c^{*}$ be a prism. If $a b b^{*} a^{*}$ has three acute angles close to $\pi / 2$, and if, moreover, the angles $\widehat{c \widehat{a O_{a b a}},}, \widehat{c b o_{a b a}}, c^{*} \widehat{a^{*} o_{a b}} a^{*}$, regarding aba*, are acute, and all analogous angles regarding $b b^{*} a^{*}, a a^{*} b^{*}, a b b^{*}$, are also acute, then the prism holds 13 discs at $a b b^{*} a^{*}$.

Proof. Indeed, all angle conditions required in Lemma 3.1 are satisfied. The condition that the angles of $a b b^{*} a^{*}$ be close to $\pi / 2$ is needed since it implies that $a b b^{*} a^{*}$ is close to a rectangle, from which $\angle b^{*} b o_{a b b^{*}}<\pi / 2$ and all other analogous inequalities follow.

A pentahedral cage, in contrast to a tetrahedral one, can hold discs not only at faces.

Consider the pyramid $P=a b c d e$ with apex $e$ and a quadrilateral face $a b c d$. If the triangle ace is acute, the capability of cage $(P)$ to hold a disc there depends on the angles $\angle b a o_{a c e}, \angle d a o_{a c e}, \angle b c o_{a c e}, \angle d c o_{a c e}, \angle b e o_{a c e}, \angle d e o_{a c e}$. If all of them are smaller than $\pi / 2$, then the pyramid holds 4 discs at ace, one on each side of $\overline{a c e}$, and two crossing $\overline{a c e}$. Here, holding a disc at ace means, in analogy to holding a disc at a face, that a certain limit disc lies in $\overline{a c e}$ (and is, in fact, circumscribed to ace). If ace is not acute, cage $(P)$ cannot hold any disc there.

Adding the at most 4 discs held at bde, we obtain a maximum of 8 held discs, which traverse the pyramid.

A (combinatorial) prism $a b c a^{*} b^{*} c^{*}$ with faces $a b c, a^{*} b^{*} c^{*}, a b b^{*} a^{*}, b c c^{*} b^{*}, c a a^{*} c^{*}$, may also hold discs at $a b c^{*}$ and at the other 5 analogous triangles. In order to hold any disc at $a b c^{*}$, we must have $\angle c c^{*} o_{a b c^{*}}<\pi / 2$ and at least one of the inequalities $\angle a^{*} c o_{a b^{*}}<$ $\pi / 2, \angle b^{*} \mathrm{co}_{a b c^{*}}<\pi / 2$. Now, if this happens, we have a held disc "separating" $a b$ from $c$ if $\angle c a o_{a b c^{*}}<\pi / 2$ and $\angle c b o_{a b c^{*}}<\pi / 2$, and a similar held disc "separating" $a b$ from $a^{*} b^{*}$ if $\angle a^{*} a o_{a b c^{*}}<\pi / 2$ and $\angle b^{*} b o_{a b c^{*}}<\pi / 2$. This amounts to a maximum of 2 discs held at $a b c^{*}$.

In particular, the following holds.

Lemma 3.4. If the prism $P$ is close to a long right regular one, then cage $(P)$ holds 2 discs at abc* and at each of the other 5 analogous places.

Moreover, Proposition 1.4 warns that there might exist limit discs not coplanar with any three vertices of the cage. Consequently, let us say that a cage $G$ holds $n$ standard discs if all corresponding end-components have limit discs coplanar with at least three vertices of conv $G$.

Thus, if $P$ is a pyramid, the total number of standard discs held by cage $(P)$ would become at most 37 , and if it is a prism at most 59 . Can these numbers be realized? Is it 59 the true maximum for all pentahedra?

But, first, let us solve Problem 1.2.

Theorem 3.5. There exists a pentahedral cage holding exactly 7 discs.

Proof. Let $Q=a b c d$ be a rectangle, of centre $o$, such that the triangles $a b o$ and $c d o$ be equilateral. Let $m$ be the centre of $a b o$. Close to $m$ choose a point $e \notin \overline{a b c}$, whose orthogonal projection on $\overline{a b c}$ is $m$. Put $o^{\prime}=o_{c d e}$. See Figure 9.

We show that, for the pyramid $P=e a b c d$, cage $(P)$ holds 7 discs.

Indeed, notice that the triangles $a b m, b c m$, and $d a m$ are obtuse. So, besides the rectangle $Q, P$ has four triangular faces, of which only cde is acute. Since $\angle a e o^{\prime}>\pi / 2$, $\angle b e o^{\prime}>\pi / 2, \angle b c o^{\prime}<\pi / 2, \angle a d o^{\prime}<\pi / 2, P$ holds 2 discs at $c d e$. 


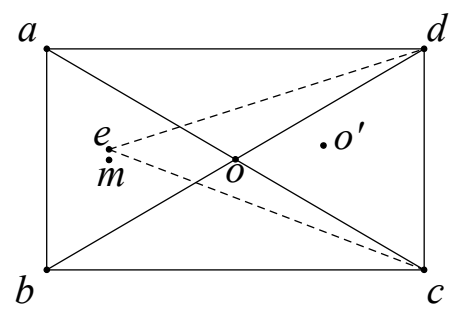

Figure 9: Cage holding 7 discs.

For the face $Q$, the relevant angles satisfy $\angle e a o=\angle e b o<\pi / 2$ and $\angle e c o=\angle e d o<$ $\pi / 2$. Hence, above all edges of $Q$ our cage holds 1 disc, while above any three of its edges and under the fourth it also holds a disc. Above any two consecutive edges of $Q$, but under the remaining two, cage $(P)$ holds no disc. Hence, it holds 5 discs at $Q$.

The two triangles $e a c$ and $e b d$ traversing $P$ are both obtuse, so no disc can be held at any of them. Clearly, there are no non-standard discs held.

In conclusion, altogether cage $(P)$ holds 7 discs, as stated.

We now establish the exact minimum for the number of discs and the exact maximum for the number of standard discs that a pentahedral cage can hold.

Three parallel lines in $\mathbb{R}^{3}$ determine an unbounded closed prism $P$ having 3 strips as sides. If a triangle $\Delta \subset \mathbb{R}^{3}$ has its vertices on the sides of $P$, we say that $P$ is associated with $\Delta$.

We shall make use of the following simple, but powerful, result.

Proposition 3.6 (Chevallier, Fruchard [5]). For any (bounded) combinatorial prism with triangular faces $\Delta$ and $\Delta^{\prime}$, it is impossible that $\Delta$ lies in the interior of a prism associated with $\Delta^{\prime}$, and $\Delta^{\prime}$ lies in the interior of a prism associated with $\Delta$.

For the reader's convenience, we give here a short proof.

Proof. Assume that $\Delta=a b c$ lies in the interior of a prism $P$ associated with $\Delta^{\prime}=a^{\prime} b^{\prime} c^{\prime}$. As $\Delta \cap \Delta^{\prime}=\emptyset$, the triangle $\Delta$ entirely lies in one component $P^{+}$of $P \backslash \overline{a^{\prime} b^{\prime} c^{\prime}}$. Thus, $\overline{a a^{\prime}}$, $\overline{b b^{\prime}}, \overline{c c^{\prime}}$ meet in some point $z \in P^{+}$. This determines the order $z, a, a^{\prime}$ on $\overline{a a^{\prime}}$. Analogously, the assumption that $\Delta^{\prime}$ lies in the interior of a prism associated with $\Delta$ implies the order $z, a^{\prime}, a$ on $\overline{a a^{\prime}}$. But both orders cannot coexist.

Lemma 3.7. For no prism $P$, cage $(P)$ can hold more than 6 discs at its triangular faces together.

Proof. Take the prism $P=a b c a^{*} b^{*} c^{*}$. We use Lemma 2.3 and its proof. We have $\angle a^{*} a o_{a b c}<\pi / 2$ if and only if $a^{*} \notin H_{a o_{a b c}}^{+}$, Hence, $\widehat{a^{*} a o_{a b} c}, \widehat{b^{*} b o_{a b c}}, \widehat{c^{*} c o_{a b c}}$ are all acute if and only if $a^{*}$ belongs to the complement of $H_{a o_{a b c}}^{+} \cup H_{b o_{a b c}}^{+} \cup H_{c o_{a b c}}^{+}$, which is the interior of a certain prism associated with $a b c$. In order for cage $\left(a b c a^{*} b^{*} c^{*}\right)$ to hold 4 discs at each of its two triangular faces, all vertices of each of them must lie in the interior of a prism associated with the other. But this is forbidden by Proposition 3.6. So, by Lemma 2.3 (adapted to our needs), cage $(P)$ cannot hold more than 6 discs at its triangular faces together. 
Theorem 3.8. A pentahedral cage can hold at least 0 and at most 57 standard discs. Both bounds are attained.

Proof. To prove that a pentahedral cage may hold no standard disc, take a trapezoid having all four triangles determined by their diagonals obtuse. A prism with such trapezoids as quadrilateral faces and with two obtuse triangles as remaining faces holds no disc, see Figure 10.
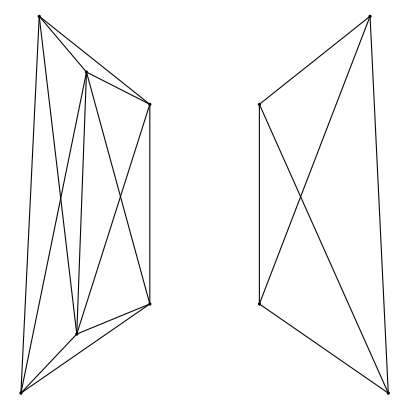

Figure 10: Cage holding no discs.

We now build a prism the cage of which holds 57 standard discs. Consider a long right regular prism $a b c a^{*} b^{*} c^{*}$ (with $a a^{*}, b b^{*}, c c^{*}$ parallel).

Choose $a_{1} \in a a^{*}$ close to $a$ and $c_{1} \in c c^{*}$ close to $c$, satisfying

$$
2\left\|a-a_{1}\right\|<\left\|c-c_{1}\right\|
$$

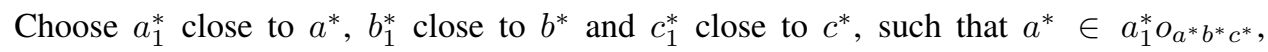
$b^{*} \in b_{1}^{*} o_{a^{*} b^{*} c^{*}}, c^{*} \in c_{1}^{*} o_{a^{*} b^{*} c^{*}}$, and

$$
\left\|a^{*}-a_{1}^{*}\right\|=\left\|b^{*}-b_{1}^{*}\right\|=\left\|c^{*}-c_{1}^{*}\right\|=\varepsilon .
$$

See Figure 11. Also, put $\left\{a^{\prime}\right\}=a a_{1}^{*} \cap \overline{a_{1} b c_{1}}$ and $\left\{c^{\prime}\right\}=c c_{1}^{*} \cap \overline{a_{1} b c_{1}}$.

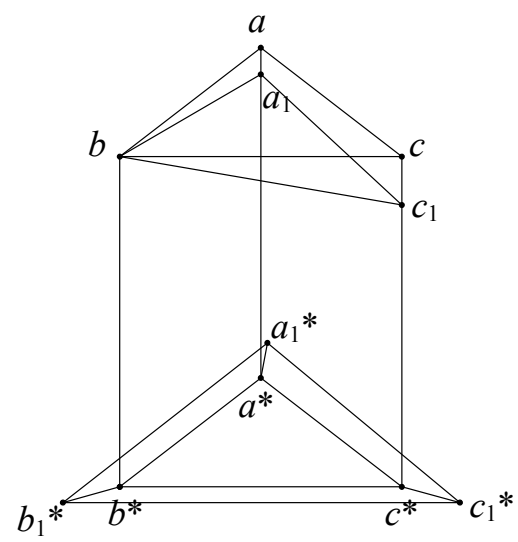

Figure 11: Cage holding 57 discs. 
If $\varepsilon$ is small enough, then the three quadrilateral faces $a^{\prime} b b_{1}^{*} a_{1}^{*}, b c^{\prime} c_{1}^{*} b_{1}^{*}, c^{\prime} a^{\prime} a_{1}^{*} c_{1}^{*}$, have obtuse angles at $a^{\prime}, c^{\prime}, c^{\prime}$, respectively, and acute angles at all other vertices.

All angles analogous to $a \widehat{b o_{b b^{*}} c^{*}}$ are acute, so they remain acute after the small changes done to $a b c a^{*} b^{*} c^{*}$. Thus, by Lemma 3.3, there are 13 discs held at each quadrilateral face.

Passing now to the two triangular faces, we immediately see that all angles $a^{\prime} \widehat{a_{1}^{*} o_{a_{1}^{*}} b_{1}^{*} c_{1}^{*}}$, $b b_{1}^{*} o_{a_{1}^{*} b_{1}^{*}} c_{1}^{*}, c^{\prime} \widehat{c_{1}^{*} o_{a_{1}^{*} b_{1}^{*}}} c_{1}^{*}$, are acute.

Concerning $a^{\prime} b c^{\prime}, \angle b^{*} b a_{1}<\pi / 2$ and $\angle b^{*} b c_{1}<\pi / 2$ imply $\angle b^{*} b o_{a_{1}} b c_{1}<\pi / 2$. Therefore, the next moves being gentle enough, $\angle b_{1}^{*} b o_{a^{\prime} b c^{\prime}}<\pi / 2$ too.

The inequality $2\left\|a-a_{1}\right\|<\left\|c-c_{1}\right\|$ yields $\angle a^{*} a_{1} o_{a_{1} b c_{1}}<\pi / 2$. Again, this can be preserved, and $\angle a_{1}^{*} a^{\prime} o_{a^{\prime} b c^{\prime}}<\pi / 2$. Now, adapting part of the proof of Lemma 2.3, we see that at least two discs are held at $a^{\prime} b c^{\prime}$. Hence, by Lemma 2.3 (see its proof) and by Lemma 3.7, our cage holds 13 discs at each of its quadrilateral faces, $4 \operatorname{discs}$ at $a_{1}^{*} b_{1}^{*} c_{1}^{*}$, and 2 discs at $a^{\prime} b c^{\prime}$.

Concerning the discs traversing the prism, the maximum number (of 12) is reached, by Lemma 3.4.

Thus, our cage holds 57 standard discs. By Lemmas 3.2 and 3.7, it cannot hold more than these 57 . The proof is finished.

Theorem 3.8 does not prove that 57 is the maximal number of discs that a pentahedral cage can hold. We miss an analogue of Proposition 2.5 for pentahedra. Examples that the referee kindly provided suggest that such an analogue may not exist. Thus, we remain with the following.

Problem 3.9. What is the maximal number of discs that a pentahedral cage can hold?

\section{References}

[1] O. Aberth, An isoperimetric inequality for polyhedra and its application to an extremal problem, Proc. London Math. Soc. 13 (1963), 322-336, doi:10.1112/plms/s3-13.1.322.

[2] I. Bárány and T. Zamfirescu, Circles holding typical convex bodies, Libertas Math. 33 (2013), 21-25, doi:10.14510/lm-ns.v33i1.47.

[3] I. Bárány and T. Zamfirescu, Holding circles and fixing frames, Discrete Comput. Geom. 50 (2013), 1101-1111, doi:10.1007/s00454-013-9549-2.

[4] A. S. Besicovitch, A cage to hold a unit-sphere, in: V. L. Klee (ed.), Convexity, American Mathematical Society, Providence, Rhode Island, volume 7 of Proceedings of Symposia in Pure Mathematics, 1963 pp. 19-20, doi:10.1090/pspum/007/0155236, held at the University of Washington, Seattle, Washington, June 13 - 15, 1961.

[5] N. Chevallier and A. Fruchard, private communication.

[6] H. S. M. Coxeter, Review 1950, Math. Reviews 20 (1959), 322.

[7] A. Fruchard, private communication.

[8] A. Fruchard and T. Zamfirescu, Cages of small length holding convex bodies, to appear, https://hal.archives-ouvertes.fr/hal-01573138.

[9] J. Itoh, Y. Tanoue and T. Zamfirescu, Tetrahedra passing through a circular or square hole, Rend. Circ. Mat. Palermo Suppl. 77 (2006), 349-354.

[10] J. Itoh and T. Zamfirescu, Simplices passing through a hole, J. Geom. 83 (2005), 65-70, doi: 10.1007/s00022-005-0013-1. 
[11] L. Montejano and T. Zamfirescu, Two problems on cages for discs, in: K. Adiprasito, I. Bárány and C. Vîlcu (eds.), Convexity and Discrete Geometry Including Graph Theory, Springer, Cham, volume 148 of Springer Proceedings in Mathematics \& Statistics, 2016 pp. 263-264, doi:10.1007/978-3-319-28186-5_24, papers from the conference held in Mulhouse, September 7 - 11, 2014.

[12] G. Valette, À propos des cages circonscrites à une sphère, Bull. Soc. Math. Belg. 21 (1969), $124-125$.

[13] T. Zamfirescu, How to hold a convex body?, Geom. Dedicata 54 (1995), 313-316, doi:10.1007/ bf01265346.

[14] T. Zamfirescu, Polytopes passing through circles, Period. Math. Hung. 57 (2008), 227-230, doi:10.1007/s10998-008-8227-8.

[15] T. Zamfirescu, Pushing convex and other bodies through rings and holes, An. Univ. Vest Timis. Ser. Mat.-Inform. 48 (2010), 299-306, http://tzamfirescu.tricube.de/ TZamfirescu-182.pdf. 